\title{
Benzocyclobutene (BCB) Based Intracortical Neural Implant
}

\author{
Kee-Keun Lee, Jiping He, Amarjit Singh, and Bruce Kim \\ Department of Bioengineering, Center for Solid State Electronics Research \\ Arizona State University, Tempe, AZ 85287-5706, USA \\ Email: keekeun@asu.edu
}

\begin{abstract}
A novel structure for chronically implantable cortical electrodes using new Benzocyclobutene (BCB) biopolymer was devised, which provides both flexibility for micro-motion compliance between brain tissues and skull and stiffness for better surgical handling. $B C B$ is very attractive polymer for stable long-term implant function, because it has flexibility, biocompatibility, low moisture uptake $(<0.2 w t \%)$, and low dielectric constant $(\sim 2.6)$.

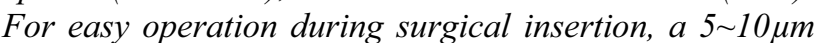
thick silicon backbone layer is attached to the desired region of the electrode to increase the stiffness. It is then followed by $1 \mathrm{~mm}$ of flexible part of the electrode without silicon backbone layer designed to absorb stress from any micro-motion between the brain tissue and the electrode. The fabricated implants have tri-shanks with 5 recording sites $(20 \times 20 \mu \mathrm{m})$ and 2 vias $(40 \times 40 \mu \mathrm{m})$ on each shank. $B C B$ electrodes with $5 \mu \mathrm{m}$ and $10 \mu \mathrm{m}$ thick backbone silicon penetrated pia of rat brain without buckling.
\end{abstract}

\section{Introduction}

Recently the ASU (Arizona State University) group reported the fabrication of polyimide-based multichannel intracortical interface and recorded the neural activities from the auditory cortex of a rat's brain [1]. This type of polymer based intracortical neural implants present several attractive features: flexible, biocompatible, and easily manufactured using existing microfabrication technology. Flexibility is highly desirable to minimize tissue damage caused by the micromotion between the brain and the implant. Unfortunately, there are some problems associated with the flexible polyimide electrodes. Due to the lack of stiffness, flexible polyimide electrodes buckle during the insertion and therefore, cannot penetrate through brain tissues for neural implant application. In addition, polyimide has relatively high water uptake (4-6 wt\%) and rough surface planarization.
Table 1. Electrical properties of BCB and PI

\begin{tabular}{ccc}
\hline Properties & BCB & PI \\
\hline Photo sensitivity type & Negative & Negative \\
Dielectric constant & 2.65 & 3.5 \\
Volume resistivity $(\mathrm{ohm} \mathrm{cm})$ & $1 \times 10^{19}$ & $10^{16}$ \\
Breakdown voltage $(\mathrm{V} / \mathrm{cm})$ & $3 \times 10^{6}$ & $3 \times 10^{6}$ \\
Glass temperature $\left({ }^{\circ} \mathrm{C}\right)$ & $>350$ & 430 \\
Water uptake $(\mathrm{wt} \%)$ & 0.12 & 4 \\
Planarization level & Excellent & Poor \\
\hline
\end{tabular}

For the first time we report on the fabrication of benzocyclobutene (BCB) polymer-based intracortical neural implant for reliable and stable long-term implant function. BCB is a very attractive polymer, because it has flexibility, biocompatibility, low moisture uptake $(<0.2$ $\mathrm{wt} \%)$, low dielectric constant ( 2.6), and excellent chemical resistant [2]. In addition, the surface of the BCB is very amenable to modifications and preparations, which allow a host of bioactive organic species to be adsorbed or covalently bonded to its surface. Therefore, the BCB electrode will enable a new type of chronically neural implant for neural recording and stimulation. Some important properties of $\mathrm{BCB}$ and polyimide (PI) are listed in Table 1 [3].

Using CMOS-compatible processing techniques, BCB-based multi-channel neural implant was fabricated. Figure 1 shows a schematic diagram of the neural implant. Pure BCB polymer-based electrode had buckling problems during insertion. For easy operation during surgical insertion into neural tissues, a $5 \sim 10 \mu \mathrm{m}$ thick silicon backbone layer, from SOI substrate, is attached to the desired region of the electrode to increase the stiffness (Young's modulus). The stiffness of the electrode can be varied by changing the thickness of the silicon backbone layer. The dimension of the stiff portion is $1.5 \mathrm{~mm}$ in length and $0.2 \mathrm{~mm}$ in width that remains inside the brain. It is then followed by $1 \mathrm{~mm}$ of flexible part of the electrode without silicon backbone layer designed to absorb stress from any micro-motion between the brain tissue and the electrode. Current microwire or silicon-based electrodes with stiff structure can cause 
additional damage to the surrounding neural or vascular tissues due to the relative micro-motion between the brain tissues and skull. Our new design can avoid these problems.

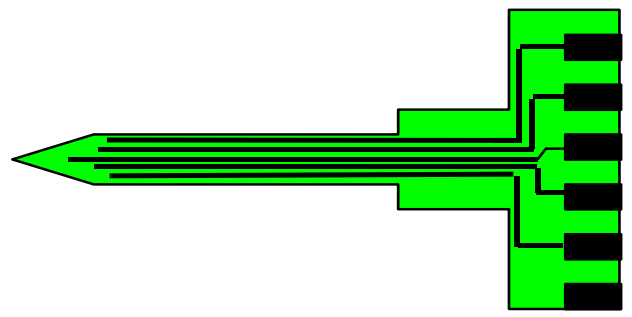

(a)

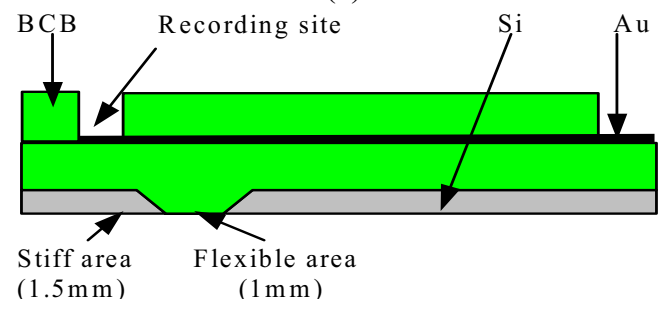

(b)

Figure 1. Simple schematic diagram of the BCB based neural implant: (a) Top view and (b) cross-sectional view.

All the recording sites were positioned near the end of the shank in order to increase the probability of recording neural signals from a target volume of tissue. The recording sites are interfaced to the external circuitry via a 15-channel connector, which is especially designed to facilitate processing of neural signals to the external circuitry. The size of the connector portion is exactly same as a 15-channel connector. The details are presented in this paper.

\section{Fabrication}

Fabrication starts with a 4-in silicon-on insulator (SOI) substrate with varying top device silicon thickness from 2 to $10 \mu \mathrm{m}$ and buried oxide thickness of $1 \mu \mathrm{m}$. Top device silicon is (100) oriented $n$-type silicon with resistivity of $10 \sim 25 \Omega-\mathrm{cm}$. SOI wafer provides easy thickness control for stiff portion and excellent etching stop during backside etch process.

Figure 2 shows schematic diagrams for fabrication procedure. Top device silicon layer was selectively etched away for flexible region using a $2000 \AA$ thick gold masking layer (Fig. 2(a)). To make smooth transition between flexible and stiff portions, silicon wet etching in 7\% Tetra Methyl Ammonium Hydroxide (TMAH) was performed at $80^{\circ} \mathrm{C}$. The silicon-etching rate depends on crystal planes in TMAH. (100) crystal plane shows 20 times faster etching rate than that of (111) plane [4]. Angled slope of the silicon removes step problem in next metallization process for recording sites, even BCB offers a degree of planarization of nearly $100 \%$ (Fig. 3). After removing gold masking layer, SOI wafer was cleaned and etched in an $80^{\circ} \mathrm{C}$, 4:1 solution of $\mathrm{H}_{2} \mathrm{SO}_{4}$ and $\mathrm{H}_{2} \mathrm{O}_{2}$. A reactive ion etch (RIE) was used to clean and microroughen the top silicon device surface prior to depositing the first BCB layer.

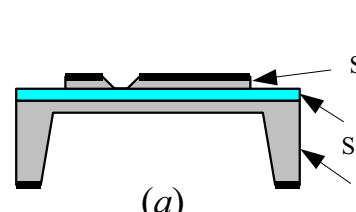

(a)

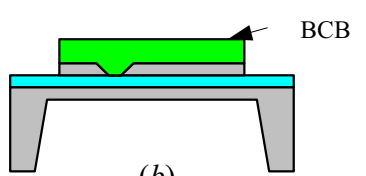

(b)

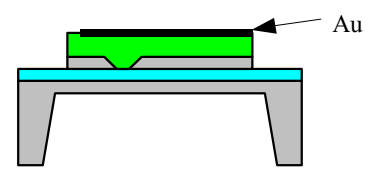

(c)

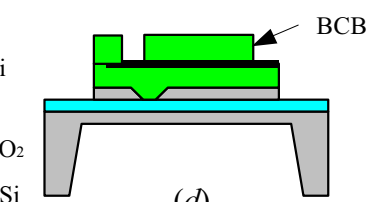

(d)

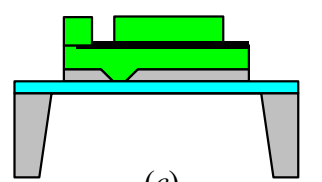

(e)

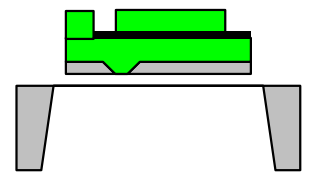

(f)
Figure 2. Fabrication procedure of the BCB electrode. (a) Selective etching of top device silicon layer for flexible region using a gold masking layer, (b) the first layer of BCB, (c) gold deposition for recording sites and gold traces, (d) the upper layer of BCB to encapsulate or reveal the desired conducting surfaces, (e) backside silicon etching, and (e) buried $\mathrm{SiO}_{2}$ etching and lifted final device.

The first layer of BCB (Cyclotene $4026^{\mathrm{TM}}$ from Dow Chemical) was spin-coated, exposed, and then developed as shown in Fig. 2(b). Then the BCB layer was partially cured for 30 minutes at $200^{\circ} \mathrm{C}$ in $\mathrm{N}_{2}$ gas environment to protect the developed pattern from subsequent processing steps and provide a suitable surface for metal deposition. Partial cure of base BCB layer and full cures of the upper $\mathrm{BCB}$ layer terminates any route for water transmission through the boundary between the base and top BCB layers. Excellent planarization and small volume shrinkage on the base BCB layer were observed.

$A$ reactive ion etch (RIE) was used to clean and micro-roughen the BCB surface. After RIE, a $2000 \AA$ thick gold layer was deposited for recording sites, followed by wet etching (Fig. 2(c)). Gold was used for recording site because it has excellent surface inertness, and it provides no native oxide. However, gold is soft material, so long-term corrosion issues should be examed. The top BCB layer was spun, exposed, and developed to encapsulate or reveal the desired conducting surfaces 
(Fig. 2(d)). The electrode was then fully cured for 1 hour at $250^{\circ} \mathrm{C}$ in $\mathrm{N}_{2}$ gas environment. The final $\mathrm{BCB}$ structures are $20 \mu \mathrm{m}$ thickness. The recording sites were not completely opened, probably due to a lateral penetration of UV light. These residues hamper signal recording and electrical contact with a 15-channel connector. RIE was used to etch away the $\mathrm{BCB}$ residue on the gold surface using a $10 \mu \mathrm{m}$ thick photoresistmasking layer. $\mathrm{A} \mathrm{CF}_{4}$ and $\mathrm{O}_{2}$ mixture was used for this descum process in RIE (100Watt, $100 \mathrm{mTorr}, 10 \mathrm{sccm} \mathrm{CF}_{4}$, and $40 \mathrm{sccm} \mathrm{O}_{2}$ ) [5].

Wafer was upside-downed for backside silicon etching in RIE. A top device surface was protected with photoresist and another dummy silicon wafer from plasma heat and RF power on the ground plane. Backside silicon etching was performed for $10 \mathrm{hrs}$ in RIE with $\mathrm{SF}_{6}(25 \mathrm{sccm}$ SF6, $5 \mathrm{sccm} \mathrm{O}$, 100mTorr, and 120Watt). Clean and uniform silicon backside etching was obtained (Fig. 2(e)). Silicon etching exactly stopped on the buried $\mathrm{SiO}_{2}$ layer due to big etching rate difference between $\mathrm{Si}$ and $\mathrm{SiO}_{2}$. After complete removal of backside silicon, the buried $\mathrm{SiO}_{2}$ was etched away in $49 \% \mathrm{HF}$ acid solution (Fig. 2(f)). Finally top-protecting photoresist was dissolved in microstripe for $2 \mathrm{hr}$ at $50^{\circ} \mathrm{C}$. Several rinses with deionized water are performed to remove any unwanted etchant products.

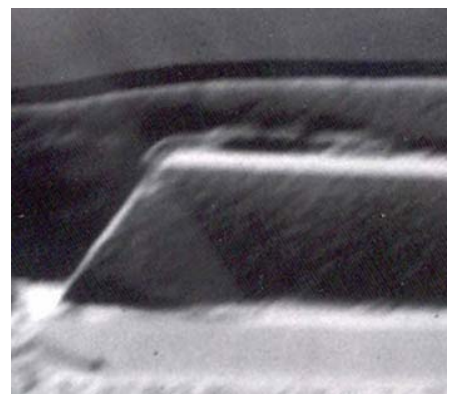

Figure 3. Cross-sectional SEM view of boundary between flexible and stiff regions. Angled slope of the silicon removes step problem in gold metallization for recording sites. BCB offers excellent planarization.

\section{Results}

The fabricated device was visualized through optical microscopy and scanning electron microscopy (SEM) as shown in Figure 4. The fabricated implants are tri-shanks with 5 recording sites $(20 \times 20 \mu \mathrm{m})$ and 2 vias $(40 \mathrm{x}$ $40 \mu \mathrm{m})$ per electrode. The stiff portion with silicon backbone layer is $1.5 \mathrm{~mm}$ in length and $0.2 \mathrm{~mm}$ in width that remains inside the brain. Flexible portion without silicon backbone layer is $1 \mathrm{~mm}$ in length. The thickness was approximately $20 \mu \mathrm{m}$. The connector portion of the completed electrode was exactly fitted into the commercial connector with 15-channel metal pads (Fig. 5).

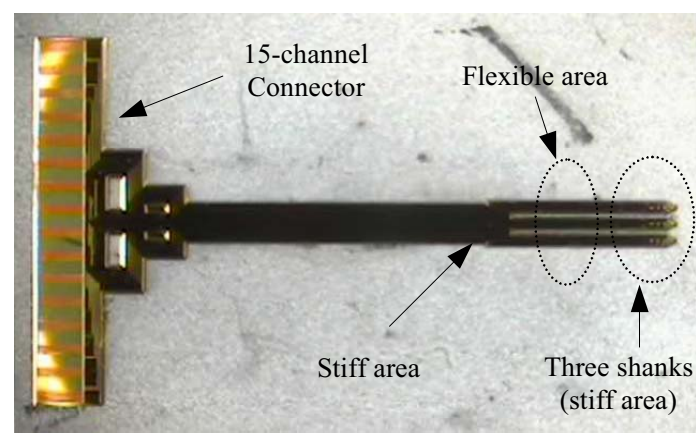

(a)

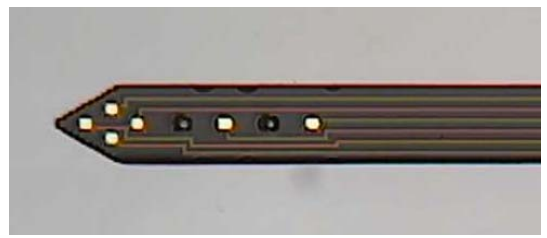

(b)

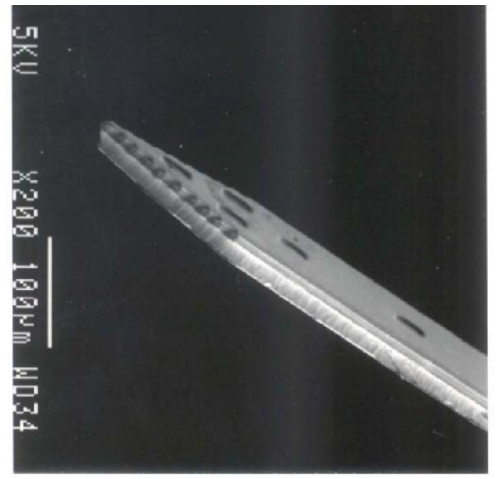

(c)
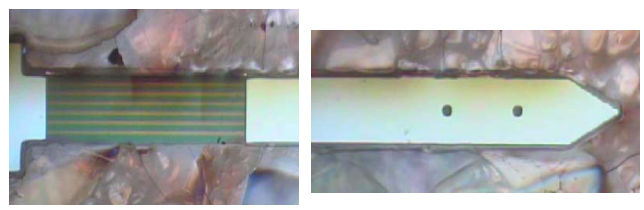

(d)

Figure 4. Optical microscope and SEM images of the fabricated electrode. (a) Entire view of the electrode, (b) top view, (c) angled view of recording sites, and (d) backside view of the electrode.

\subsection{Electrical test}

Electrical impedance testing was performed using HP 4284A precision LCR meter. All the recording sites were immersed into a $0.9 \%$ saline solution at room temperature [6]. When an alternating current source is passed through 
one of the recording sites and the saline solution, a potential develops at the electrode-electrolyte interface. This potential determines the recording site impedance. The area of the recording site was $400 \mu \mathrm{m}^{2}$. 3 devices were tested. The frequency was varied from $100 \mathrm{~Hz}$ to $10 \mathrm{KHz}$, while the alternating peak-to-peak current source was set at 50,100, and $200 \mu \mathrm{A}$.

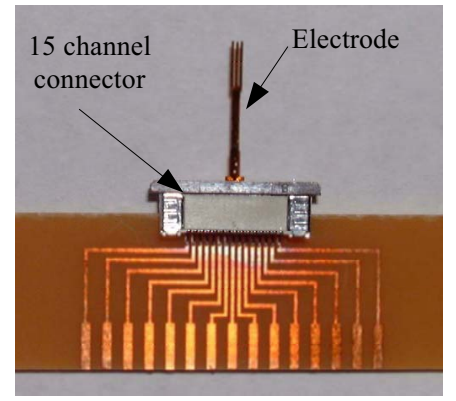

Figure 5. Microscopic image of the connector and electrode. The connector portion of the completed probe was exactly fitted into the commercial connector with 15 channel metal pads.

From 3 devices at $100 \mu \mathrm{A}$ peak-to-peak current, the averaged impedance value at $1 \mathrm{KHz}$ was $\sim 2 \mathrm{Mohm}$. The impedance remained stable over several weeks because of excellent water protection in the $\mathrm{BCB}$ dielectric layers, and partial cure of base BCB layer and full cures of the upper BCB layer. This curing approach terminates any route for water transmission through the boundary between the base and top BCB layers. Figure 6 shows impedance values depending on the peak-to-peak alternating test current sources. There is no any significant dependency on the testing current sources. The size and material of the recording sites mainly control impedance values.

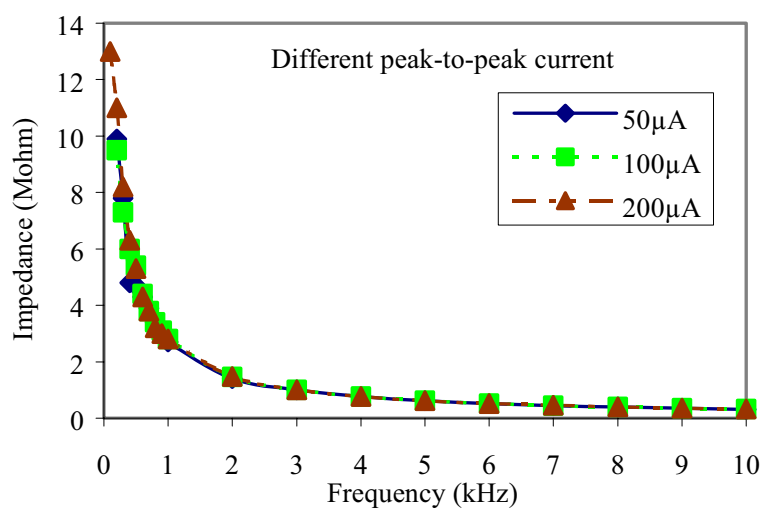

Figure 6. Measured impedance values in terms of frequencies. Three different peak-to-peak current were tested at one $20 \times 20 \mu \mathrm{m}$ recording site.

\subsection{Mechanical test}

Mechanical stiffness test of the stiff portion of the electrode shank was performed using micro force thermomechanical tester. For this test, pure BCB and silicon electrodes were also fabricated, as shown in Fig. 7. Electrode strength and stiffness are closely depending on the probe geometry such as width, length and thickness [7]. In a condition where all other parameters are same, the stiffness was tested in terms of the different silicon backbone thickness. The underneath backbone silicon thickness was varied from $2 \mu \mathrm{m}$ to $10 \mu \mathrm{m}$. The probe dimensions are $1.5 \mathrm{~mm} \times 0.2 \mathrm{~mm} \times 0.02 \mathrm{~mm}$, in condition where there is no silicon backbone layer.

The fabricated electrode was placed in the testing machine and a stretching force was applied until probe breaks. An extensometer was used to measure the amount that the electrode stretches between the gage marks. We convert the force to stress and the distance between gage marks to strain. The young's modulus is the slope of the stress-strain curve in the elastic region and the measure of the stiffness [8].

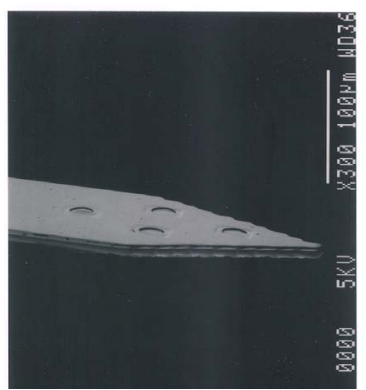

(a)

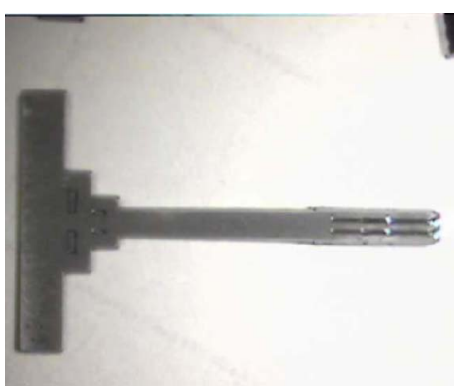

(b)

Figure 7. SEM images of (a) pure BCB electrode without silicon backbone layer and (b) pure silicon-based electrodes for penetration test.

For a $5 \mu \mathrm{m}$ thick silicon backbone BCB electrode, the stiffness was improved to 32Gpa. This increase is 10 times larger than that of the electrode without silicon backbone layer. It was further shown that the stiffness could be increased to $60 \mathrm{Gpa}$ with a thicker layer $(10 \mu \mathrm{m})$ of silicon as shown in Table 2 . 


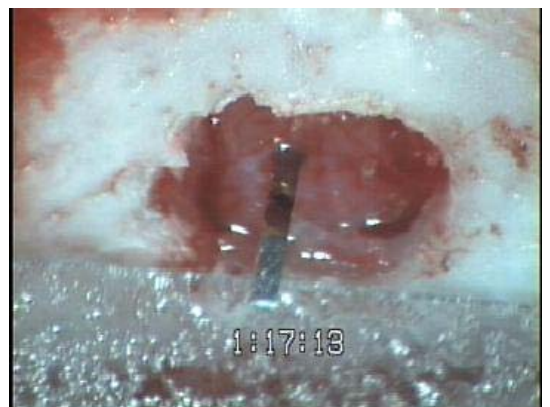

Figure 8. Optical microscope picture of the penetration test into rat's pia. $5 \mu \mathrm{m}$ thick silicon backbone BCB electrode penetrates pia without buckling.

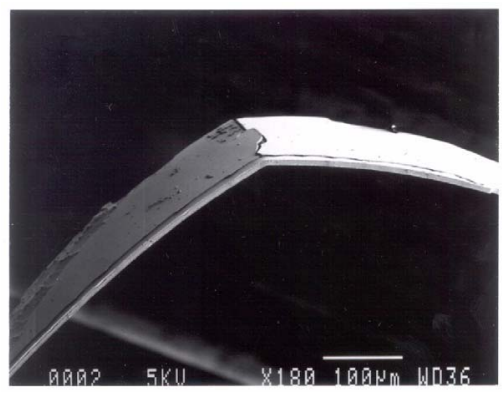

Figure 9. SEM picture with $2 \mu \mathrm{m}$-thick broken backbone silicon. Electrode was fractured during insertion into the pia.

Table 2. Measured mechanical stiffness (Young's

\begin{tabular}{ccc}
\multicolumn{3}{c}{ modulus) values } \\
\hline & $\begin{array}{c}\text { Young's } \\
\text { modulus } \\
(\mathrm{GPa})\end{array}$ & $\begin{array}{c}\text { Rat pia } \\
\text { penetration }\end{array}$ \\
\hline $\begin{array}{c}\text { Pure BCB electrode } \\
2 \mu \mathrm{m} \mathrm{Si} \mathrm{backbone} \\
\text { electrode }\end{array}$ & 3 & No \\
$5 \mu \mathrm{m} \mathrm{Si}$ & 32 & No \\
$10 \mu \mathrm{m} \mathrm{Si}$ & 60 & Yes \\
Bulk Si & 110 & Yes \\
\hline
\end{tabular}

Penetration test into rat's brain was performed to check whether the microprobe could penetrate the pia and dura without any surgery aid tool and buckling. For the test, rat was anaesthetized and heart rate and oxygen saturation were monitored. Skull and dura were removed and the stiff electrode was lowered to the surface (pia) by hand. Great care was made to encourage post implant recovery. Enough force was applied using Teflon tweezer. Stiff electrodes with $5 \mu \mathrm{m}$ and $10 \mu \mathrm{m}$ thick backbone silicon penetrated pia of rat without buckling (Fig. 8). A $2 \mu \mathrm{m}$ thick silicon backbone electrode was fractured during an insertion into the pia, in which $\mathrm{BCB}$ film was not damaged, as shown in Fig. 9. Testing results are summarized in Table 2.

\section{Conclusion}

For the first time BCB polymer-based intracortical neural implant was fabricated for reliable and stable longterm implant function. Pure BCB electrode had buckling problems during insertion. For easy operation during surgical insertion, a $5 \sim 10 \mu \mathrm{m}$ thick silicon backbone layer was attached to the desired region of the electrode to increase the stiffness. It is then followed by $1 \mathrm{~mm}$ of flexible part of the electrode without silicon backbone layer designed to absorb stress from any micro-motion between the brain tissue and the electrode. For the biocompatibility test, $\mathrm{BCB}$ was evaluated to be non reactive and non cytoxic. The area of the recording sites was $400 \mu \mathrm{m}^{2}$. The averaged impedance value at $1 \mathrm{KHz}$ was $\sim 2$ Mohm. The impedance remained stable over several weeks because of excellent water protection in the $\mathrm{BCB}$ dielectric layers, and partial cure of base $\mathrm{BCB}$ layer and full cures of the upper BCB layer. For a $5 \mu \mathrm{m}$ thick silicon backbone electrode, the stiffness was improved 10 times larger than that of the electrode without silicon backbone layer. In penetration test into rat's pia, stiff electrodes with $5 \mu \mathrm{m}$ and $10 \mu \mathrm{m}$ thick backbone silicon penetrated pia of rat without buckling, while a $2 \mu \mathrm{m}$ thick silicon backbone electrode was fractured before creating an insertion into the pia.

\section{Acknowledgments}

The authors wish to acknowledge the support of the DARPA /Bio:Info:Micro program.

\section{References}

[1] P. Rousche, D. Pellinen, D. Pivin, J. Williams, R. Vetter, and D. Kipke, "Flexible polyimide-based intracortical electrode arrays with bioactive capability," IEEE Trans. Biomedical Eng., Vol. 48, No. 3, pp. 361, 2001.

[2] A. Strandjord, W. Rogers, and S. Cummings, "MCM-D fabrication with photosensitive benzocyclobutene," Proceedings of ISHM, pp. 402, 1995.

[3] M. Ohnmacht, V. Seidemann, and S. Buttgenbach, "Microcoils and microrelays," Sensors and Actuators A, Vol. 83, pp. 124, 2001.

[4] O. Tabata, "Anisotropic Etching of Si in TMAH solutions," Sensors and Materials, Vol. 13, No. 5, pp. 271, 2001.

[5] C. Korman, T. Chow, and D. Bower, "Etching characteristics of polysilicon, $\mathrm{SiO}_{2}$ and $\mathrm{MoSi}_{2}$ in $\mathrm{NF}_{3}$ and $\mathrm{SF}_{6}$ plasmas," Solid State Tech., pp. 115, January 1983.

[6] N. Blum, B. Carkhuff, H. Charles, R. Edwards, and R. Meyer, "Multisite microprobes for neural recordings," IEEE Trans. Biomedical Eng., Vol. 38, No. 1, pp. 68, 1991.

[7] K. Najafi and J. Hetke, "Strength characterization of silicon microprobes in neurophysiological tissues," IEEE Trans. Biomedical Eng., Vol. 37, No. 5, pp. 474, 1990.

[8] D. Askeland, The science and engineering of materials, PWS-KENT Publishing Company, 1989. 